Document downloaded from:

http://hdl.handle.net/10251/70564

This paper must be cited as:

Martí Calatayud, MC.; García Gabaldón, M.; Pérez-Herranz, V.; Sonia; SERGIO MESTRE (2013). Chronopotentiometric study of ceramic cation-exchange membranes based on zirconium phosphate in contact with nickel sulfate solutions. Desalination and Water Treatment. 51(1):597-605. doi:10.1080/19443994.2012.714629.

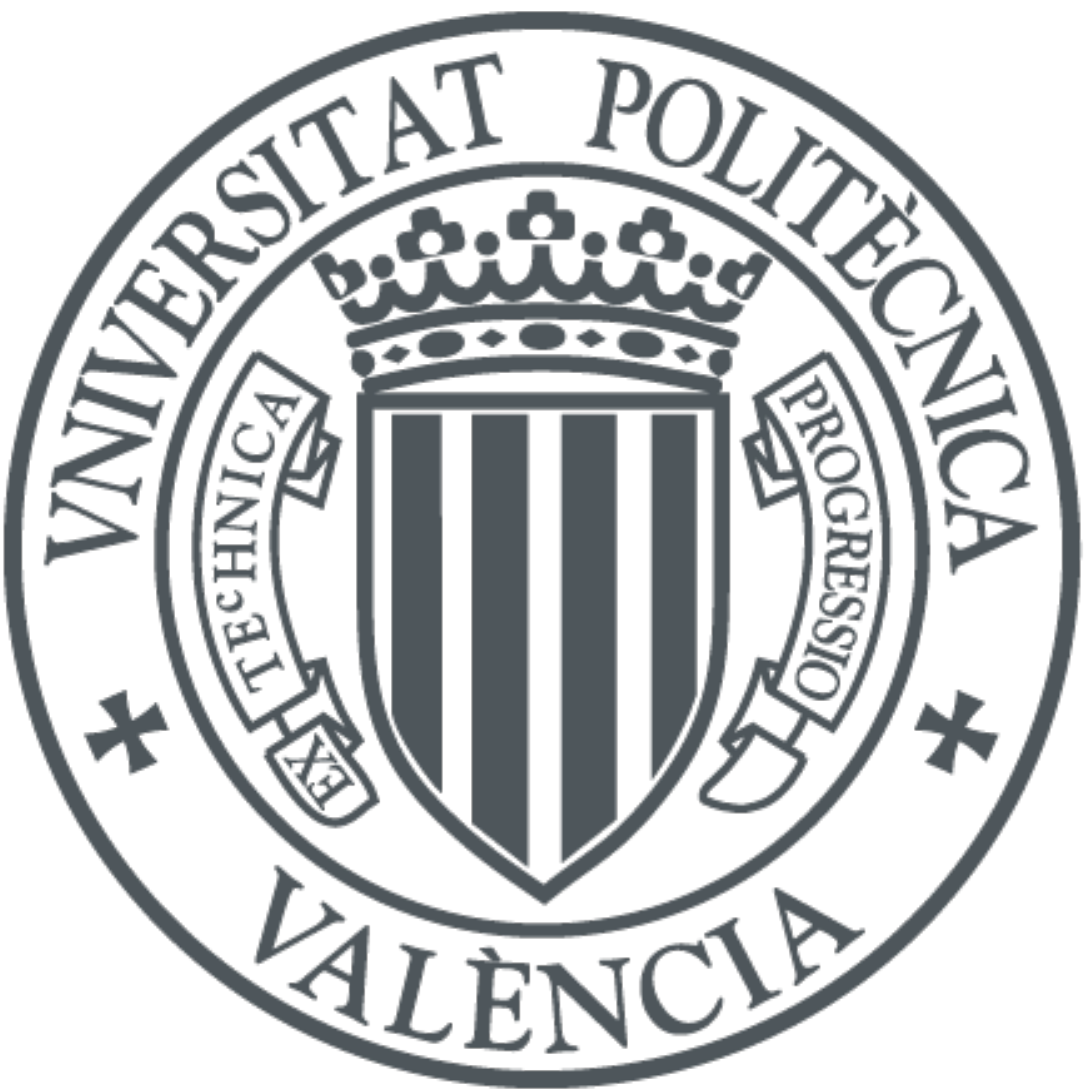

The final publication is available at

http://dx.doi.org/10.1080/19443994.2012.714629

Copyright Taylor \& Francis

Additional Information 


\title{
Chronopotentiometric study of ceramic cation-exchange membranes based on zirconium phosphate in contact with nickel sulfate solutions.
}

\author{
M.C. Martí-Calatayud ${ }^{\mathrm{a} *}$, M. García-Gabaldón ${ }^{\mathrm{a}}$, V. Pérez-Herranz ${ }^{\mathrm{a}}, \mathrm{S}_{\text {S Sales }}^{\mathrm{b}}$, \\ S. Mestre ${ }^{\mathrm{b}}$ \\ ${ }^{a}$ IEC Group, Departamento de Ingeniería Química y Nuclear, E.T.S.I. Industriales, Universitat Politècnica de \\ València, PO Box 22012, E-46071 València, Spain \\ Tel.+34 963877632; Fax:+34963877639; email: mamarc13@upvnet.upv.es \\ ${ }^{b}$ Instituto de Tecnología Cerámica, Campus Universitario Riu Sec, Av. Vicent Sos Baynat s/n, 12006 Castellón, \\ Spain
}

*Corresponding author. email: mamarc13@upvnet.upv.es

\begin{abstract}
.
In this article, the innovative cation-exchange membranes obtained from ceramic materials are presented. Different microporous ceramic supports were obtained from an initial mixture of alumina and kaolin, to which a varying content of starch was added in order to obtain supports with different pore size distributions. The deposition of zirconium phosphate into the porous supports generates membranes with cation-exchange properties. The fabrication of ionexchange membranes which could resist aggressive electrolytes such as strong oxidizing spent chromium plating baths or radioactive solutions would allow the application of electro-dialysis for the decontamination and regeneration of these industrial effluents. The perfor- mance of the manufactured membranes was studied in nickel sulfate solutions by means of chronopotentiometry. An increase of the membrane voltage drop during chronopotentiometric measurements was observed in some membranes, which seems to be a consequence of concentration polarization phenomena resulting from the ionic transfer occurred through the membranes. Current-voltage curves were obtained for the different ceramic membranes, allowing the calculation of their ohmic resistance. The ohmic resistance of the membranes increased when the open porosity (OP) of the samples was incremented up to a value of $50 \%$. For values of OP higher than $50 \%$, the resistance of the membranes decreased significantly with porosity.
\end{abstract}

Keywords: Ceramic cation-exchange membranes; Chronopotentiometry; Zirconium phosphate; Electrodialysis; Industrial waste water reuse 


\section{Introduction.}

The use of large volumes of water associated with industrial processes constitutes an important part of the total consumption of the available water resources. Specially, in several manufacturing processes, a final rinse stage in which important volumes of water are used is necessary for the cleaning of the manufactured objects and its final presentation. Specifically, in the case of metal finishing industries a rinse cascade step follows the chromium plating [1], which is applied in order to impart corrosion resistance and esthetic properties to metallic pieces. The spent rinse waters generated in chromium plating industries contain important amounts of hexavalent chromium present in the composition of the plating baths. Moreover, during the plating process the concentration of different metallic cations $\mathrm{Ni}^{2+}$, $\mathrm{Al}^{3+}, \mathrm{Cu}^{2+}$, and $\mathrm{Fe}^{3+}$ ) builds up in the process baths, being then transferred to the rinse waters. Consequently, a significant amount of chromic acid is wasted and its direct recirculation to the plating process is inadvisable due to the presence of cationic contaminants which would imply an important decrease of the current efficiency. Besides this, the carcinogenic effects of hexavalent chromium and the oxidizing and acidic nature of these effluents entail a great environmental concern. At the same time, the management of spent rinse waters without a further reuse in the process involves an important waste of water and raw materials.

The selective removal of cationic metals which contaminate the spent baths (such as $\mathrm{Ni}^{2+}$ ions) is feasible by means of using cation-exchange membranes (CEMs) in electrodialysis cells. Thus, the recirculation of the concentrated chromic acid to the process tank would lead to a major efficiency in the utilization of chromic acid for its initial purpose, whereas spent rinse waters can be decontaminated and reused with an important reduction of the overall water requirements for the process. However, the main drawback of electrodialysis is the reduced chemical stability of ion-exchange membranes against oxidizing, acidic, and radioactive media. Most of the commercial ion-exchange membranes are made of polymeric materials, which mostly cannot bear the extreme conditions of specific industrial effluents. Moreover, the high cost of polymeric ion-exchange membranes with improved chemical resistance restrains its utilization for such applications. Therefore, the manufacture of ion- exchange membranes made exclusively of inexpensive ceramic materials, which could resist oxidizing solutions, will lead to an increasing application of electro- dialysis operations for certain situations in which commercial polymeric ion-exchange membranes are too expensive or have a short durability. In this vein, previous studies have proven the suitability of ceramic ion-exchange 
materials for the treatment of radioactive waste waters [2,3].

With the aim of obtaining ion-exchange membranes from ceramic materials, distinct procedures have been followed in previous studies [4-6]. Among them, the manufacture of composite CEMs for their use in direct methanol fuel cells stands out due to the reduced methanol permeability and increased proton conductivity offered by those membranes [7]. In most of these membranes, the ion-exchange material is based on zirconium phosphate or its derivatives, being the proton exchange of these acid salts basically determined by the layered structure of their crystals. The $\alpha-\mathrm{HZrP}$ crystals $\left(\mathrm{Zr}(\mathrm{HPO} 4) 2 \cdot \mathrm{H}_{2} \mathrm{O}\right)$ are formed by refluxing the amorphous gels of $\mathrm{Zr}$ (IV) salts in phosphate-containing solutions [8]. Each phosphate has one oxygen atom bearing a proton oriented toward the interlayer space which is exchangeable with the electrolyte cations. Apart from the interlayer protons, exchangeable with those cations susceptible to enter in the interlayer cavities, the surface crystals of $\alpha-\mathrm{HZrP}$ are also known to own interesting properties of ion exchange, especially with large cations of transition elements [9]. It is also believed that the exchange process in layered compounds is much faster at their surface than in the bulk material, being this caused by an increased presence of vacant structural sites [10-12].

The energetic requirement of an electrochemical reactor is another important factor which deserves special attention. In this case, the electric resistance of ion-exchange membranes is a parameter which affects significantly the total energy consumption in the treatment of waste effluents by means of electrochemical reactors. The voltage drop through the separator is one of the main contributions to the total cell voltage. The variation of the electric conductance of the porous supports as a function of the initial composition of the membranes has been studied in a previous work [5]. In that work, it was proven that the porosimetric parameters such as the open porosity (OP) or pore size distribution can be modified by means of the inclusion of an organic compound in the raw materials mixture of the ceramic supports.

The cation-exchange properties of the surface of HZrP crystals are considered in the present work in order to evaluate their inclusion in a ceramic support. In this sense, the use of host materials as a support for guest molecules which provide the whole structure with specific properties has been applied in several fields [13]. In the present case, microporous ceramic structures formed by a matrix derived from alumina and kaolin are used as the porous support, whereas the active ion exchanger should provide the desired ion-exchange properties. Different manufacturing parameters of the membranes are varied in order to obtain supports with diverse microstructures. The porosimetric parameters are determined for the 
different supports and ion-exchange membranes, since the average pore diameter and the pore size distribution are supposed to be key parameters in the effective deposition of zirconium phosphate and the resulting membrane properties. Chronopotentiometric experiments are then performed in order to evaluate the transport properties of the different membranes. Chronopotentiometry is a useful technique which aids in the investigation of different phenomena taking place in interfacial systems. It allows in the case of a membrane/electrolyte system the study of interfacial mass transfer processes as well as the determination of important parameters for energetic considerations such as the resistance of the membrane and the adjoining solution layers. 


\section{Experimental.}

\subsection{Materials and reagents}

Synthetic solutions of nickel sulfate with a concentration of $10^{-3}$ and $10^{-2} \mathrm{M}$ were prepared with dis- tilled water and $\mathrm{NiSO} 4 \cdot 6 \mathrm{H} 2 \mathrm{O}(99 \%$, purchased from Panreac). The $\mathrm{Ni}^{2+}$ ions were selected as the target ions whose transport through the membranes will be evaluated since they are one of the main cationic contaminants present in chromium plating baths. The anionexchange membrane (AEM) used in the experiments was a polymeric one made from vinyl monomer and acrylic fiber (Ionics AR-204-SZRA-412, from Ionics Inc.). This membrane was used in order to avoid any influence of the $\mathrm{H}^{+}$ions generated at the anode on the measurement of the reference electrodes placed near the membrane under study.

\subsection{Synthesis of the membranes}

The porous supports were synthesized from a mix- ture at 50\% in weight of alumina (AR12B5, Aluminium Pechiney, France) and kaolin (ER, Caobar S.A., Spain), with variable additions of starch (starch from potatoes, Fluka AG, Germany), as a pore former. The proportions were designed to produce a mixture that was feasible to shape by dry pressing. The raw materials were homogenized in a ball mill with acetone. The resulting suspension was dried under IR lamps and moistured to a water content of $4 \mathrm{~kg} \mathrm{H} 2 \mathrm{O} / 100 \mathrm{~kg}$ dry solid. Cylindrical test specimens were formed by uniaxial dry pressing at $400 \mathrm{~kg} \mathrm{~cm}^{-2}$. Then, the green supports were dried in an oven at $110^{\circ} \mathrm{C}$ and sintered in an electric kiln (RHF1600, Carbolite Furnaces Ltd., UK), with a thermal cycle designed to oxidize the organic matter before reaching the 6 $\mathrm{h}$ permanence at maximum temperature $\left(1,400^{\circ} \mathrm{C}\right)$.

The cation exchanger was deposited in the porous network of the supports by direct precipitation. Firstly, the ceramic supports were partially immersed for $30 \mathrm{~min}$ in a $0.5 \mathrm{M} \mathrm{ZrOCl} 2 \cdot 8 \mathrm{H}_{2} \mathrm{O}$ solution $(98 \%$, Sigma Aldrich $\mathrm{GmbH}$, Germany), so that only the lower surface of the supports was in contact with the liquid and the solution was sucked into the porous network. Secondly, the solution saturated supports were immersed in H3PO4 (85\%, Fluka AG, Germany) for $24 \mathrm{~h}$ to precipitate zirconium phosphate inside the pores. Thirdly, the membranes were washed with distilled water to neutral reaction and dried at $110^{\circ} \mathrm{C}$. Finally, the membranes were thermally treated at $450^{\circ} \mathrm{C}$ in order to fix the zirconium phosphate particles on the surface of the pores. The infiltration process was repeated up to six times in the compositions with $20 \%$ of starch so as to determine the effect of the amount of zirconium phosphate deposited into the porous network. 
The experimental cell used for the chronopotentiometric measurements consists of three compartments of $130 \mathrm{ml}$ of volume each one, between which the AEM and the CEM were clamped, as seen in Fig. 1. Two graphite bars were placed at the side compartments and were used as working and counter electrodes. A potentiostat/galvanostat (Autolab, PGSTAT 20) was responsible for the energy supply between both the electrodes and the voltage drop across the membrane under study $(U \mathrm{~m})$ was measured by means of two different reference $\mathrm{Ag} / \mathrm{AgCl}$ electrodes immersed in Luggin capillaries. The system was connected to a data acquisition system in order to register the obtained results in a computer. Prior to each experiment, both membranes were equilibrated during $24 \mathrm{~h}$ under conditions of random stirring in the solutions to be used subsequently. The experiments firstly consisted of obtaining the current-voltage curve of each ceramic membrane by means of conducting a current sweep voltammetry. Then, chronopotentiometric tests were performed by means of applying various current pulses for each membrane. During the first $300 \mathrm{~s}$ of current pulse duration, a constant value of current is imposed between both the graphite electrodes while the voltage drop across the membrane was registered. Then, the current is switched off and the relaxation of the system is allowed during 300 additional seconds. 


\section{Results and discussion}

\subsection{Membranes characterization}

Table 1 shows the OP and the mean pore size (d50) values of the supports and the membranes impregnated with zirconium phosphate as a function of the content of starch present in the initial mixture and the number of impregnations with zirconium phosphate (which has been denoted as $n$ ). The pore size distribution, which is shown in Fig. 2 for different membranes, was bimodal for the supports obtained from starch contents lower than $10 \%$ but changed to trimodal for higher contents of starch because the pores created by the starch particles were progressively interconnected. After the infiltration process, the porosity was reduced by the zirconium phosphate deposited in the porous network, as it was expected. However, its distribution was not homogeneous. Firstly, it seemed that zirconium phosphate particles were deposited preferably in the pores of lower diameter (Fig. 2). Secondly, the zirconium phosphate accumulates in some areas of the supports near the surfaces, as seen from Fig. 3.

\subsection{Chronopotentiometric curves}

Chronopotentiometric curves of the different porous supports obtained from varying contents of starch are presented in Fig. 4. The porous supports without zirconium phosphate (Fig. 4(a)) show a plane chronopotentiometric response, with a shape similar to that of the corresponding applied current pulse. Firstly, at the beginning of each pulse when the current is switched on, an immediate increase of the membrane voltage drop appears in the chronopotentiometric curve. This initial voltage drop is directly related to the ohmic overvoltage of the system due to the ohmic resistance offered by the membrane and the solution layers adjacent to it $(\eta \Omega)[14,15]$. During the rest of the pulse, the registered voltage shows no time delay with respect to the applied current and remains constant until the current is switched off. This reveals the absence of important concentration polarization effects and confirms that the behavior of the porous supports corresponds to an ohmic pattern. Finally, when the current is interrupted the voltage drop reaches immediately zero. In the case of the ceramic membranes obtained from supports with different starch contents and submitted to one zirconium phosphate impregnation cycle (Fig. 4(b)), a slight increase in the $U_{\mathrm{m}}$ values is observed during the application of the current pulse in some cases depending on the values of applied current and starch content. The increase in the voltage drop occurring during the application of the current pulse is known as the total overvoltage $(\eta T)$, which is attributed to the interfacial transfer overvoltage $(\eta t)$ and the concentration overvoltage $(\eta c)$. Though being very small in comparison with the ohmic contribution, the appearance 
of $\eta_{\mathrm{T}}$ confirms the effect of $\mathrm{HZrP}$ on the interfacial transfer due to the exchange of $\mathrm{Ni}^{2+}$ ions. However, since the main contribution to the final value of $U_{\mathrm{m}}$ is that of $\eta \Omega$, a prevailing ohmic dependence would also describe the behavior of the membranes obtained after one impregnation cycle.

In the case of membranes submitted to more than two impregnation cycles (see Fig. 5), when certain values of current are applied (e.g. $i \geq 0.31 \mathrm{~mA} / \mathrm{cm}^{2}$ in Fig. 5) higher increases of the membrane voltage drop take place during the current pulse. In contrast to the results observed previously, the concentration polarization phenomenon taking place in the proximity of the membrane surface is not negligible and the contribution of the interfacial mass transfer taking place at the membrane/solution interface becomes evident. Moreover, as the current increases the contribution of the interfacial transfer overvoltage rises considerably. This improved transfer of ions through the membranes occurs as a consequence of the increased ion transport numbers in the membrane phase with respect to those in the bulk solution, thus revealing the positive effect of the impregnation of the ceramic supports with zirconium phosphate. Therefore, the ion-exchange process taking place due to the active ion-exchanger influences the behavior of these membranes and the ohmic conductance cannot be the only process describing the current-voltage dependence of the membranes. It is also to be noted that when the values of applied current are considerably increased, the chronopotentiometric response shows a decline after the first increase of $U \mathrm{~m}$. This could be originated by the occurrence of water splitting near the membrane and the passage of $\mathrm{H}^{+}$ions through the membrane when high values of current are imposed.

The chronopotentiometric results for the membranes obtained after several zirconium phosphate impregnation cycles reveal an increased participation of the interfacial transfer in the total response of the membranes, which is quite similar to the response observed in previous studies with commercial polymeric ion-exchange membranes [16-18]. For the comparison of the chronopotentiometric response of the membranes obtained in the present work with that of commercial ones, a plot of the chronopotentiograms of different membranes is presented in Fig. 6. If the typical response of a polymeric membrane is considered, a sharp increase in the membrane voltage drop occurs following the registration of the initial ohmic overvoltage. This sharp increase is considered to be a consequence of the depletion of counterions at the membrane surface in the diluate compartment taking place when currents higher than the limiting value are imposed [17]. Then, the voltage drop reaches a steady value and finally diminishes drastically when the cur- rent ceases. Nonetheless, in some cases $U m$ does 
not diminish directly to zero and a relaxation profile of the voltage drop is observed during a few seconds. The overpotential registered immediately after the current interruption is named as the concentration overvoltage $(\eta \mathrm{c})$, which is part of $\eta \mathrm{T}$. Now, if the response of the ceramic porous support without zirconium phosphate is considered, a plane response of the membrane voltage drop with a shape analogous to that of the current pulse is observed. This is consistent with the ohmic behavior of the supports and also resembles the results of previous studies conducted with ceramic membranes $[5,6,19]$. On the contrary, the ceramic membranes obtained after several impregnation cycles show the important increase in $U \mathrm{~m}$ above mentioned. However, the increase in $U_{\mathrm{m}}$ takes place more gradually than that observed with commercial polymeric membranes. Therefore, the response of the ceramic membranes can be considered as intermediate between that shown by the ceramic supports and that of commercial polymeric membranes. This could be due to the occurrence of two different mechanisms of mass transfer through the membrane phase as a con-sequence of its microporous structure: the ion exchange occurring at the pore walls due to the properties of $\mathrm{HZrP}$ and the bulk migration taking place through the internal axial volume of the largest pores. In addition to this, the significantly high thickness of the ceramic membranes $(\cong 0.6 \mathrm{~cm})$ in comparison with that of polymeric ones may also affect the different obtained responses.

\subsection{Concentration polarization: ohmic and interfacial transfer overvoltages.}

As commented previously, chronopotentiometry is a useful technique which gives insight into the interpretation of different mass transfer phenomena taking place in a membrane/electrolyte interface. Besides this, it allows the separation of the total membrane voltage drop resulting from the application of an electric field across an ion-exchange membrane into several contributions. Firstly, as a consequence of the application of an electric field an ohmic resistance arises, which is related to the $\eta \Omega$ value calculated from the initial value of $U_{\mathrm{m}}$ registered during the application of a current pulse. Then, the fact that an electrochemical separator would have ion-exchange properties can be evaluated from the further evolution of $U \mathrm{~m}$, as explained in the previous section. Thus, if the concentration polarization becomes evident, a notable increase in $U_{\mathrm{m}}$ during the course of a current pulse may be noted. The difference between the last and the first value of $U \mathrm{~m}$ of a current pulse constitutes the total overvoltage $(\eta T)$ which should be significantly higher than zero if concentration polarization takes part in the process. This approach is used in the present section in order to determine the influence of the impregnation with zirconium phosphate and the electrolyte concentration on the transport properties of the manufactured 
membranes.

The contribution of $\eta \Omega$ and $\eta \mathrm{t}$ to the final value of $U_{\mathrm{m}}$ obtained in $10^{-3} \mathrm{M}$ NiSO4 solutions for a ceramic support without impregnation and an impregnated membrane is presented in Fig. 7. In the case of the porous support, the evolution of $U_{\mathrm{m}}$ and $\eta \Omega$ with the applied current is overlapped, thus confirming an ohmic relationship between current and voltage drop. On the other hand, the contribution of the interfacial transfer overvoltage is negligible. With regard to the ceramic membranes with zirconium phosphate, at high values of current density, the contribution of $\eta t$ increases significantly, which denotes the limiting transfer phenomena associated with the increased transport of ions by means of the ion exchange taking place with HZrP. Moreover, from the plot it is seen that the ohmic overpotential follows a straight dependence with the current density, whereas the increase in $\eta \mathrm{t}$ implies a bending in the curve of $U \mathrm{~m}$. The bending results in a kind of second region in the current-voltage curve in which very little increments in the applied current density result in high increases in the membrane voltage drop. Finally, for the highest values of applied current $U \mathrm{~m}$ values decrease due to the passage of water splitting products.

Fig. 8 shows the plot of $U \mathrm{~m}, \eta \Omega$, and $\eta \mathrm{t}$ values obtained in $10^{-2} \mathrm{M}$ NiSO4 solutions for a support and a membrane with $n=3$. With regard to the contribution of the interfacial transfer and ohmic overpotentials to the total membrane voltage drop, the results are analogous to those shown for more diluted solutions. Zirconium phosphate imparts ion-exchange properties to the ceramic supports. These properties entail increased nickel transport numbers in the membrane with respect to those in the bulk solution, which promotes a limiting transfer of ions to the membrane surface due to concentration polarization. However, the limiting transfer seems to be diminished with an increase in the electrolyte concentration. Indeed, the deviation of $U \mathrm{~m}$ from the ohmic behavior followed by $\eta \Omega$ is very slight with $10^{-2} \mathrm{M}$ NiSO4 solutions, being the contribution of $\eta \mathrm{t}$ very small in this case.

\subsection{Current-voltage curves and membrane resistance}

The current-voltage curves obtained from the current sweep voltammetry experiments for membranes with different number of impregnations in $10^{-2} \mathrm{M}$ NiSO4 solutions are presented in Fig. 9. First of all, the curves of supports and different starch contents confirm a slight decrease of the resistance with the addition of starch to the raw materials composition, 
which is a consequence of the increased values of OP. Moreover, the impregnation of the porous supports several times leads to a further reduction in the slope of the curves, as seen in Fig. 9 for membranes with $n=2$ and $n=3$. Increasing zirconium phosphate infiltration cycles entail a significant blockage of pores with a consequent increase of the membrane resistance. With regard to the concentration polarization effects for the membranes submitted to two and three impregnation cycles, a change of slope is observed with the bending of the current-voltage curve when the applied current reaches a certain value. Attending to the shape of the chronopotentiograms commented before, the change of the slope in the current-voltage curves may be due to concentration polarization effects arising as a consequence of the ion exchange of $\mathrm{Ni}^{2+}$ ions facilitated by the zirconium phosphate deposited on the pore walls of the membranes.

Taking into account the first region of the current-voltage curves for which the membranes have a quasi-ohmic behavior, the electric resistance of each membrane $(R \mathrm{~m})$ can be calculated from the inverse of the slope of the curve. In Fig. 10, the relationship between the starch content and the values of OP and resistance is shown for supports $(n=0)$ and mem- branes with $n=1$. The results shown in Fig. 10 were obtained with $10^{-2} \mathrm{M}$ NiSO4 solutions, being the results corresponding to $10^{-3} \mathrm{M}$ NiSO4 solutions analogous. For the supports, the ohmic resistance diminishes with the addition of starch, which coincide with the increase in the mean pore size and OP values. The decrease in porosity and mean pore size values as a consequence of the deposited zirconium phosphate leads to a general increase of the ohmic resistance for the membranes in relation with the support employed in their synthesis. This confirms the blockage of pores resulting from the deposition of zirconium phosphate particles. However, in the case of the membranes, the dependence of $R \mathrm{~m}$ with the starch content is not descending in every case. A maximum in $R \mathrm{~m}$ is found for the support with a starch content of $10 \%$ whereas for larger starch contents, which coincide with OP values higher than $50 \%$, the support resistance decreases significantly. For starch contents lower than or equal to $10 \%$, the supports have a bimodal pore size distribution, and the increase in the OP values implies the presence of more pores being susceptible of impregnation (Fig. 2). On the contrary, the pore size distribution of the support changes from bimodal to trimodal for high starch contents with the appearance of a fraction of pores of about 3 $\mu \mathrm{m}$. This change means not only a higher volume of electrolyte inside the membrane structure, but also the interconnection of pores generated by the starch particles, which results in a significant reduction of the membrane resistance. Therefore, the pore size distribution seems to be responsible for the maximum in $R \mathrm{~m}$. 
The ohmic resistance and OP values obtained for the ceramic membranes in $10^{-3} \mathrm{M} \mathrm{NiSO} 4$ solutions are represented in Fig. 11 as a function of the number of impregnation cycles. These results confirm the close correlation between $\mathrm{OP}$ and membrane resistance. The increase in the number of impregnation cycles to which the microporous supports were submitted leads to a higher blockage of pores and this is translated into a reduction of the volume of electrolyte present in the membrane structure. This turns into a sharp increase of $R \mathrm{~m}$ values with increasing values of $n$. 


\section{Conclusions}

The CEMs obtained exclusively from ceramic materials have been presented in this work. Effects on the pore size distribution and the electrochemical behavior of the addition of starch into the initial alumina-kaolin mixture and the inclusion of zirconium phosphate particles in the porous network have been studied by means of mercury intrusion porosimetry and chronopotentiometry, respectively. The addition of starch allows the manufacture of ceramic supports with increased mean pore size and OP values. This turns into a reduction of the electric resistance values. On the other hand, the impregnation with zirconium phosphate particles provokes the blockage of internal pores and increases the membrane resistance. However, in terms of ion-exchange properties, the application of several zirconium phosphate impregnation cycles generates ceramic membranes with improved selectivity toward cations. This is reflected by an increase in the membrane voltage drop observed in the chronopotentiometric curves which is directly related to the interfacial mass transfer. The resulting chronopotentiometric response of these impregnated supports is similar to that of commercial ion-exchange membranes which results in the appearance of a second region after the ohmic one in the current-voltage curves of the membranes.

The development of ceramic ion-conducting membranes is a promising field of research since it would allow the application of electrodialysis for the treatment of industrial waste effluents with aggressive characteristics, thus allowing the reuse of expensive raw materials in industrial operations, the recycling of water, and the reduction of contaminated effluents generated by the industries. 
M.C. Martí-Calatayud would like to express his gratitude to Universitat Politè cnica de València for a postgraduate grant (Ref: 2010-12). S. Sales would like to express her gratitude to Ministerio de Ciencia e Investigación (Spain) for a postgraduate grant (AP2009-4409). This work was supported by Ministerio de Ciencia e Investigación (Spain) with the project numbers CTQ2008-06750-C02-01/PPQ and CTQ2008-06750-C02-02/PPQ 


\section{References}

[1] L. Harttinger, Handbook of Effluent Treatment and Recycling for The Metal Finishing Industry, Finishing Publications Ltd.; ASM International, Stevenage 1994.

[2] S. Balagopal, T. Landro, S. Zecevic, D. Sutija, S. Elangovan, A. Khandkar, Selective sodium removal from aqueous waste streams with NaSicon ceramics, Sep. Purif. Technol. 15 (1999) 231-237.

[3] D.T. Hobbs, Caustic recovery from alkaline nuclear waste by an electrochemical separation process, Sep. Purif. Technol. 15 (1999) 239-253.

[4] Y.S. Dzyazko, A. Mahmoud, F. Lapicque, V.N. Belyakov, Cr (VI) transport through ceramic ion-exchange membranes for treatment of industrial wastewaters, J. Appl. Electrochem. 37 (2007) 209-217.

[5] M. García-Gabaldón, V. Pérez-Herranz, E. Sánchez, S. Mestre, Effect of porosity on the effective electrical conductivity of different ceramic membranes used as sep- arators in eletrochemical reactors, J. Membr. Sci. 280 (2006) 536-544.

[6] V.M. Linkov, V.N. Belyakov, Novel ceramic membranes for electrodialysis, Sep. Purif. Technol. 25 (2001) 57-63.

[7] B.P. Tripathi, V.K. Shahi, SPEEK-zirconium hydrogen phosphate composite membranes with low methanol permeability prepared by electro-migration and in situ precipitation, J. Col- loid Interface Sci. 316 (2007) 612-621.

[8] A. Clearfield, G.D. Smith, The crystallography and structure of a-zirconium bis(monohydrogen orthophosphate) monohydrate, Inorganic Chem. 8 (1969) 431-436.

[9] G. Alberti, M.G. Bernasconi, M. Casciola, U. Costantino, Ion exchange of some divalent and trivalent cations on the sur- face of zirconium acid phosphate micro-crystals, J. Chromatogr. A 160 (1978) 109-115.

[10] A.B. Yaroslavtsev, Ion diffusion throw interface in heterogeneous solid systems with the modified surface, Defect Diffu- sion Forum 216-217 (2003) 133-140.

[11] A.B. Yaroslavtsev, Composite materials with ionic conductivity: From inorganic composites to hybrid membranes, Russ. Chem. Rev. 78 (2009) 10131029.

[12] A.B. Yaroslavtsev, V.V. Nikonenko, V.I. Zabolotsky, Ion transfer in ionexchange and membrane materials, Russ. Chem. Rev. 72 (2003) 393-421.

[13] M.E. Davis, Ordered porous materials for emerging applications, Nature 417 (2002) 813-821.

[14] M. Taky, G. Pourcelly, F. Lebon, C. Gavach, Polarization phenomena at the interfaces between an electrolyte solution and an ion exchange membrane: Part I. Ion transfer with a cation exchange membrane, J. Electroanalytical Chem. 336 (1992) 171-194.

[15] P. Sistat, G. Pourcelly, Chronopotentiometric response of an ion-exchange membrane in the underlimiting current-range. Transport phenomena within the 
diffusion layers, J. Membr. Sci. 123 (1997) 121-131.

[16] N. Pismenskaia, P. Sistat, P. Huguet, V. Nikonenko, G. Pourcelly, Chronopotentiometry applied to the study of ion transfer through anion exchange membranes, J. Membr. Sci. 228 (2004) 65-76.

[17] M.C. Martí-Calatayud, M. García-Gabaldón, V. Pérez-Herranz, E. Ortega, Determination of transport properties of $\mathrm{Ni}$ (II) through a Nafion cationexchange membrane in chromic acid solutions, J. Membr. Sci. 379 (2011) 449458.

[18] M. García-Gabaldón, V. Pérez-Herranz, E. Ortega, Evaluation of two ionexchange membranes for the transport of tin in the presence of hydrochloric acid, J. Membr. Sci. 371 (2011) 65-74.

[19] M. García-Gabaldó n, V. Pérez-Herranz, E. Sánchez, S. Mestre, Effect of tin concentration on the electrical properties of cera- mic membranes used as separators in electrochemical reac- tors, J. Membr. Sci. 323 (2008) 213-220. 
TABLES.

Table 1. Characteristics of the supports and the membranes.

\begin{tabular}{llllllll}
\hline $\begin{array}{l}\text { Starch } \\
(\text { wt.\%) }\end{array}$ & $n$ & $\begin{array}{l}\text { OP } \\
(\%)\end{array}$ & $\begin{array}{l}d_{50} \\
(\mu \mathrm{m})\end{array}$ & $\begin{array}{l}\text { Starch } \\
(\text { wt. } \%)\end{array}$ & $n \begin{array}{l}\text { OP } \\
(\%)\end{array}$ & $\begin{array}{l}d_{50} \\
(\mu \mathrm{m})\end{array}$ \\
\hline 0 & 0 & 42.8 & 0.27 & 20 & 0 & 55.67 & 0.53 \\
& 1 & 39.7 & 0.23 & & 1 & 51.00 & 0.53 \\
5 & 0 & 47.1 & 0.33 & & 2 & 48.80 & 0.53 \\
& 1 & 43.4 & 0.32 & & 3 & 47.65 & 0.45 \\
10 & 0 & 49.6 & 0.34 & & 4 & 46.54 & 0.50 \\
& 1 & 46.4 & 0.32 & & 5 & 42.84 & 0.53 \\
& & & & & 6 & 41.82 & 0.50 \\
15 & 0 & 53.2 & 0.44 & 25 & 0 & 54.5 & 0.62 \\
& 1 & 50.5 & 0.44 & & 1 & 51.4 & 0.67 \\
\hline
\end{tabular}


FIGURES.

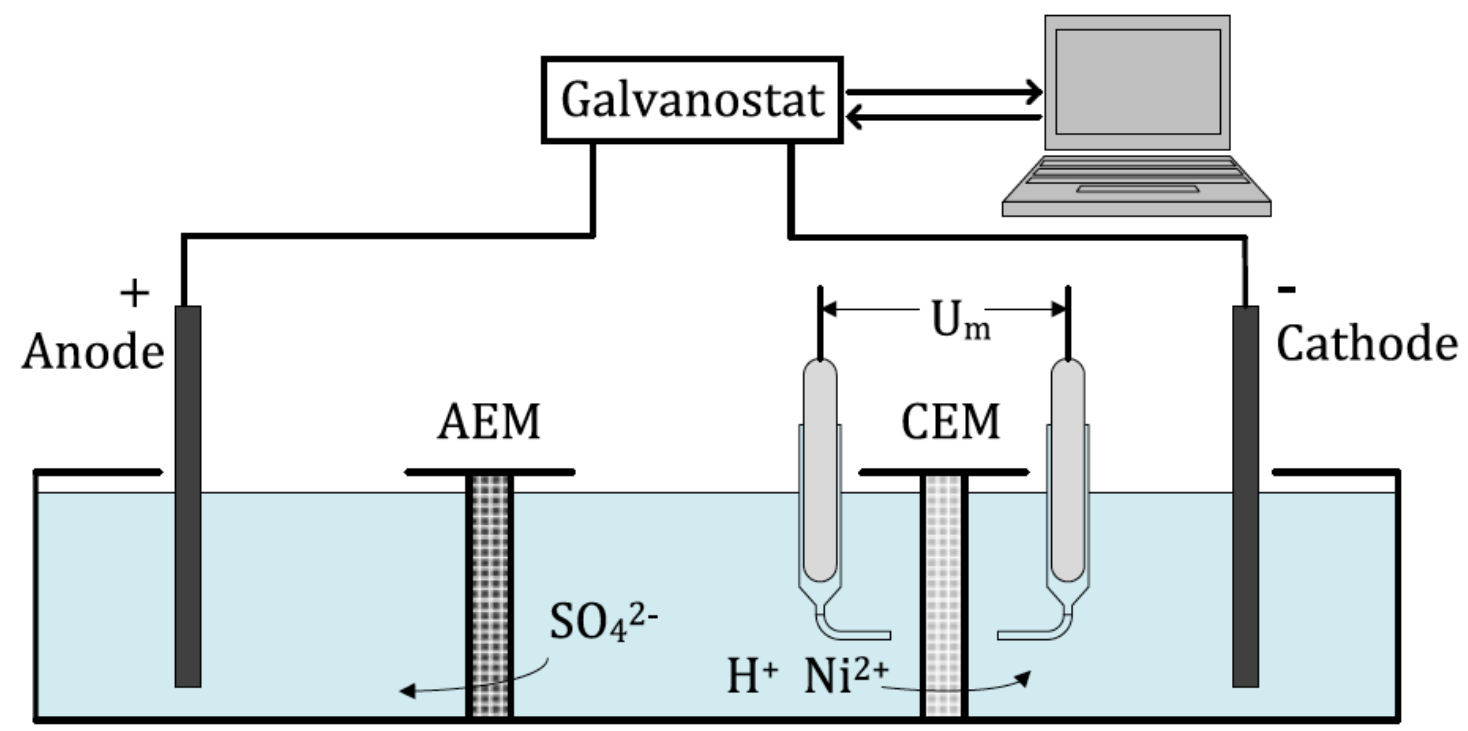

Figure 1. Experimental setup used for the chronopotentiometric experiments.

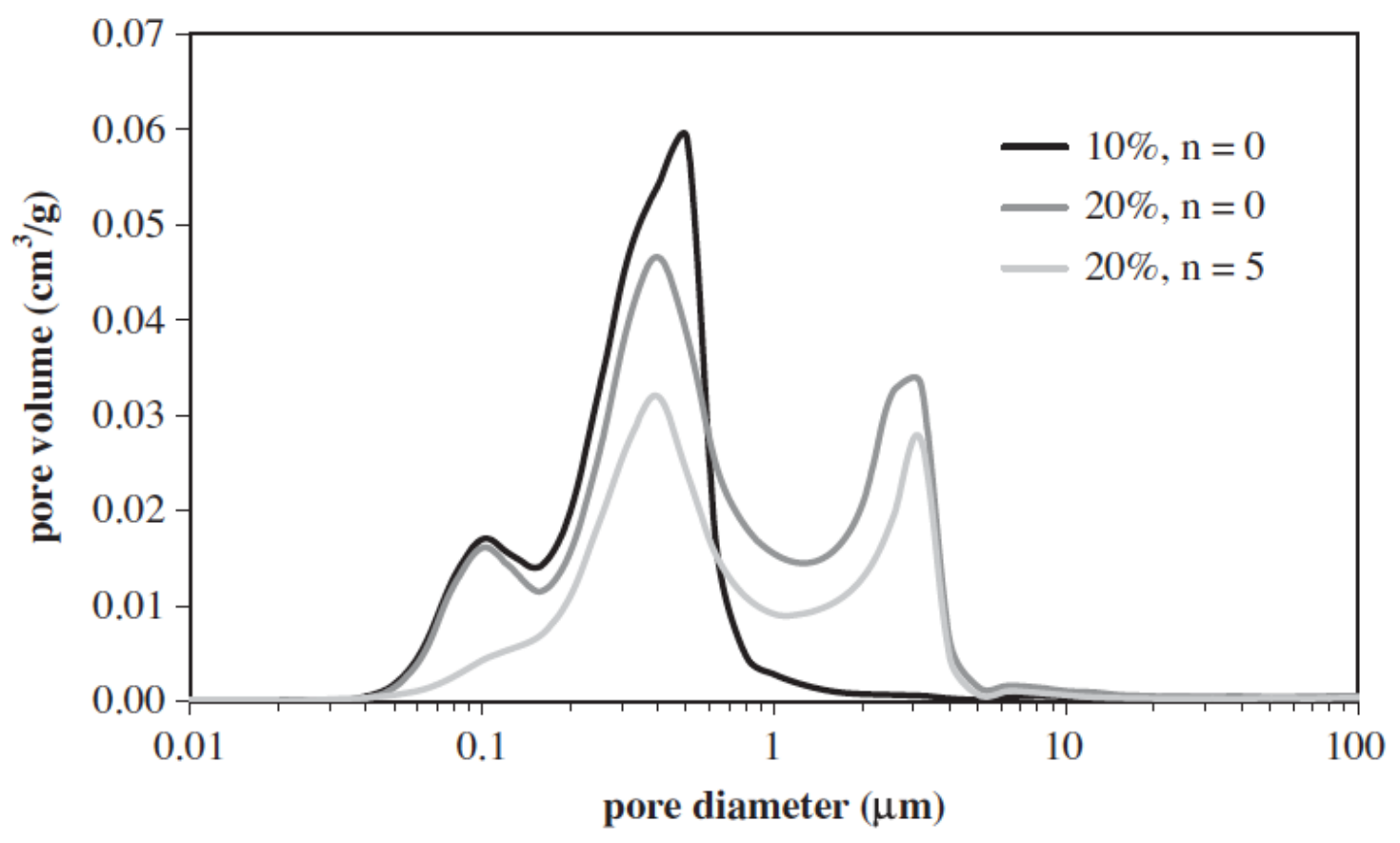

Figure 2. Pore size distributions of two supports (10\% and $20 \%$ of starch) and one membrane after being impregnated $(20 \%$ of starch and $n=5)$. 


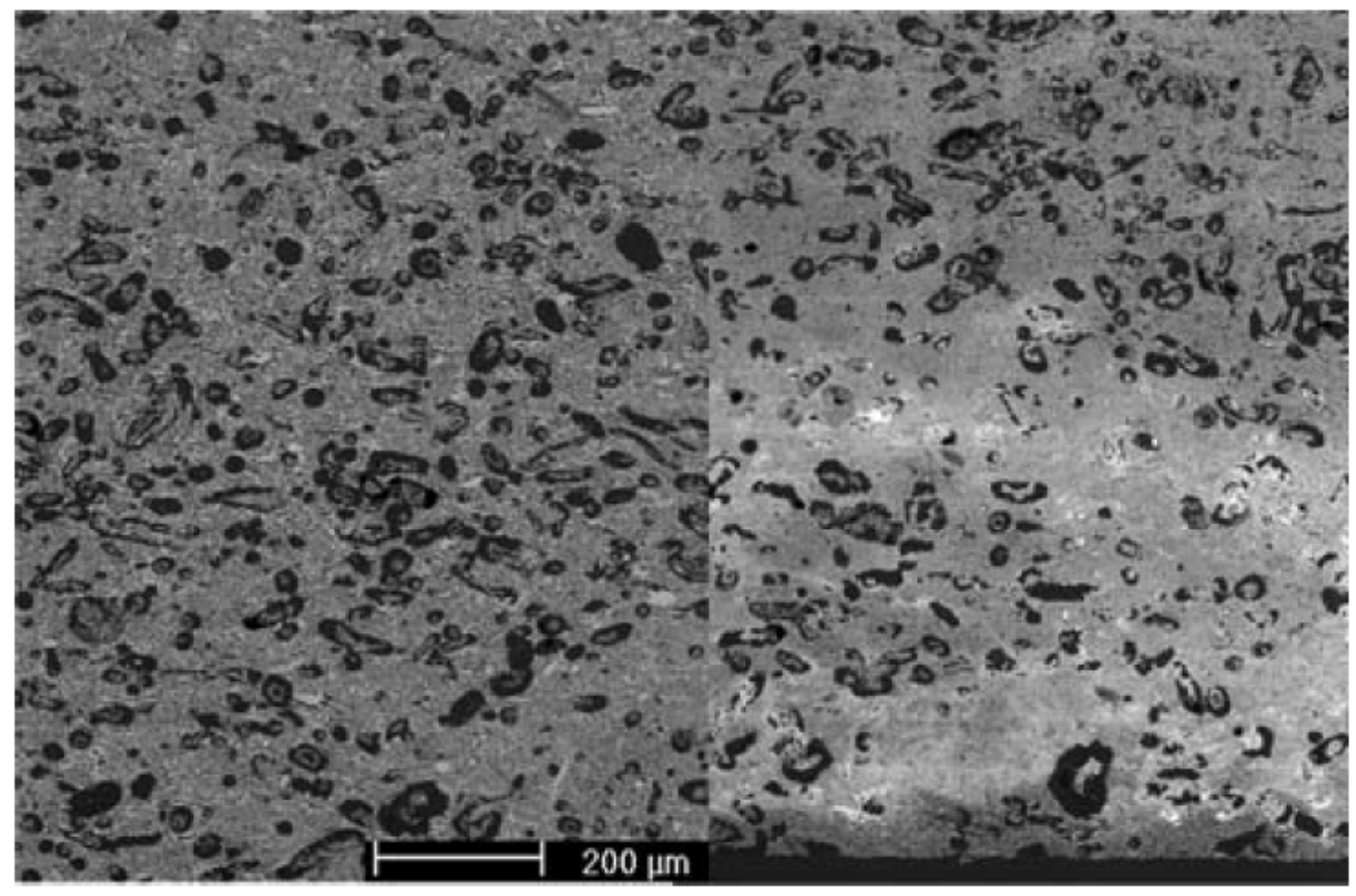

Figure 3. Scanning electron microscope images of polished sections. Left: support obtained with $20 \%$ of starch. Right: membrane obtained after five cycles of infiltration from a support with $20 \%$ of starch. 

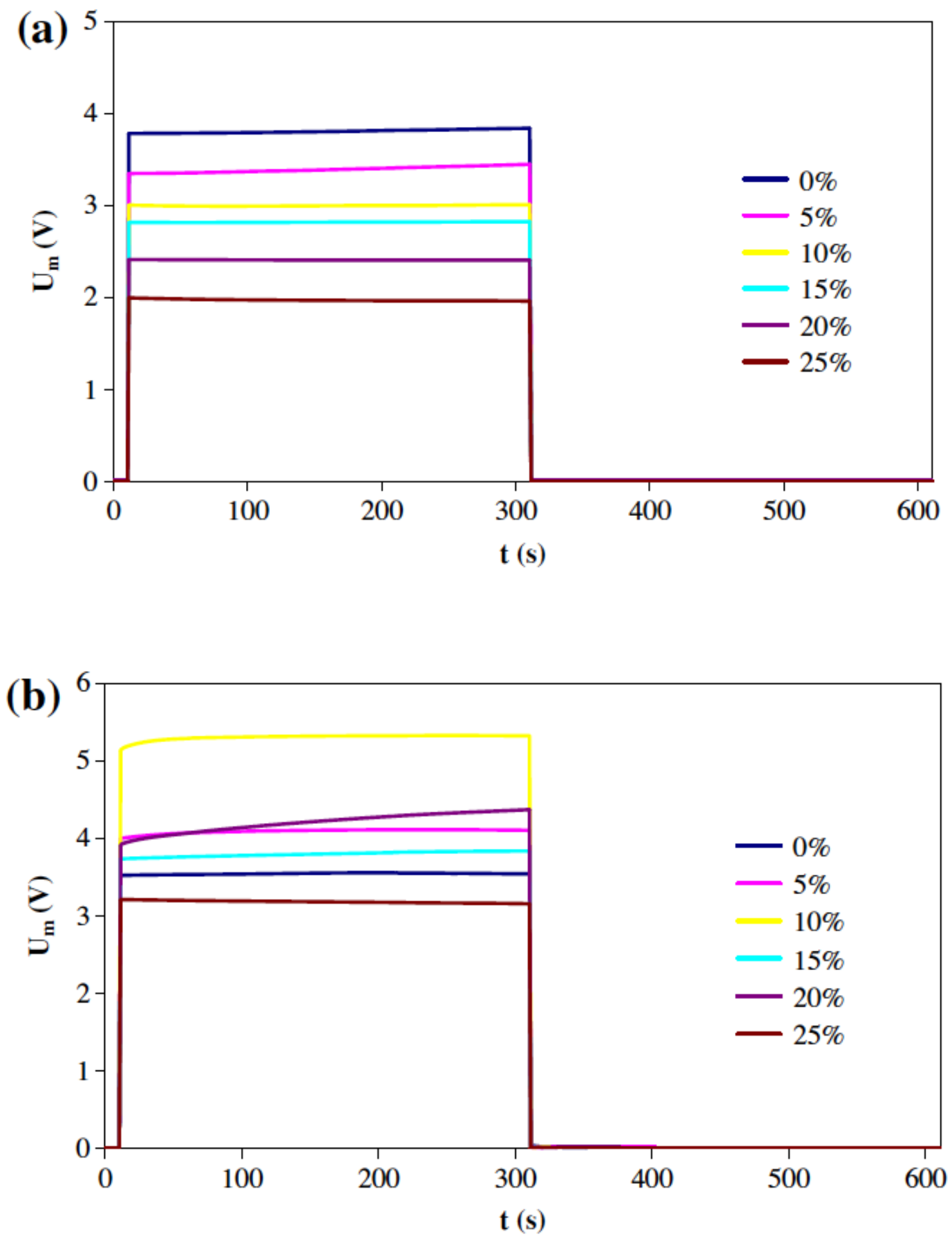

Figure 4. Chronopotentiometric response of ceramic membranes with (a) $n=0$ and (b) $\mathrm{n}=1$ in $10^{-3} \mathrm{M} \mathrm{NiSO}_{4}$ solutions under the imposition of current pulses of $0.31 \mathrm{~mA} / \mathrm{cm}^{2}$. 


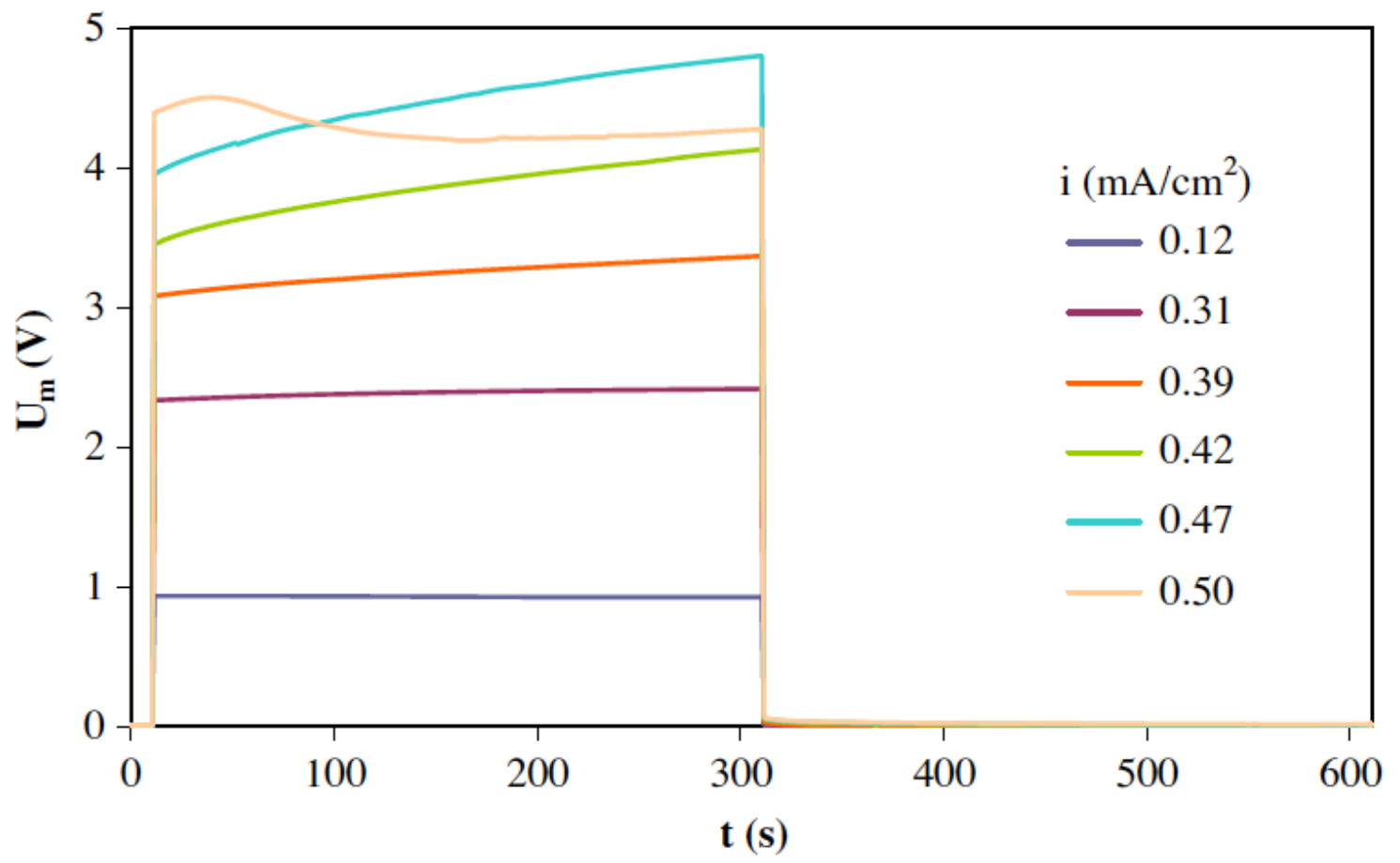

Figure 5. Chronopotentiometric response of the ceramic membranes with $n=4$ in $10^{-3} \mathrm{M} \mathrm{NiSO}_{4}$ solutions.

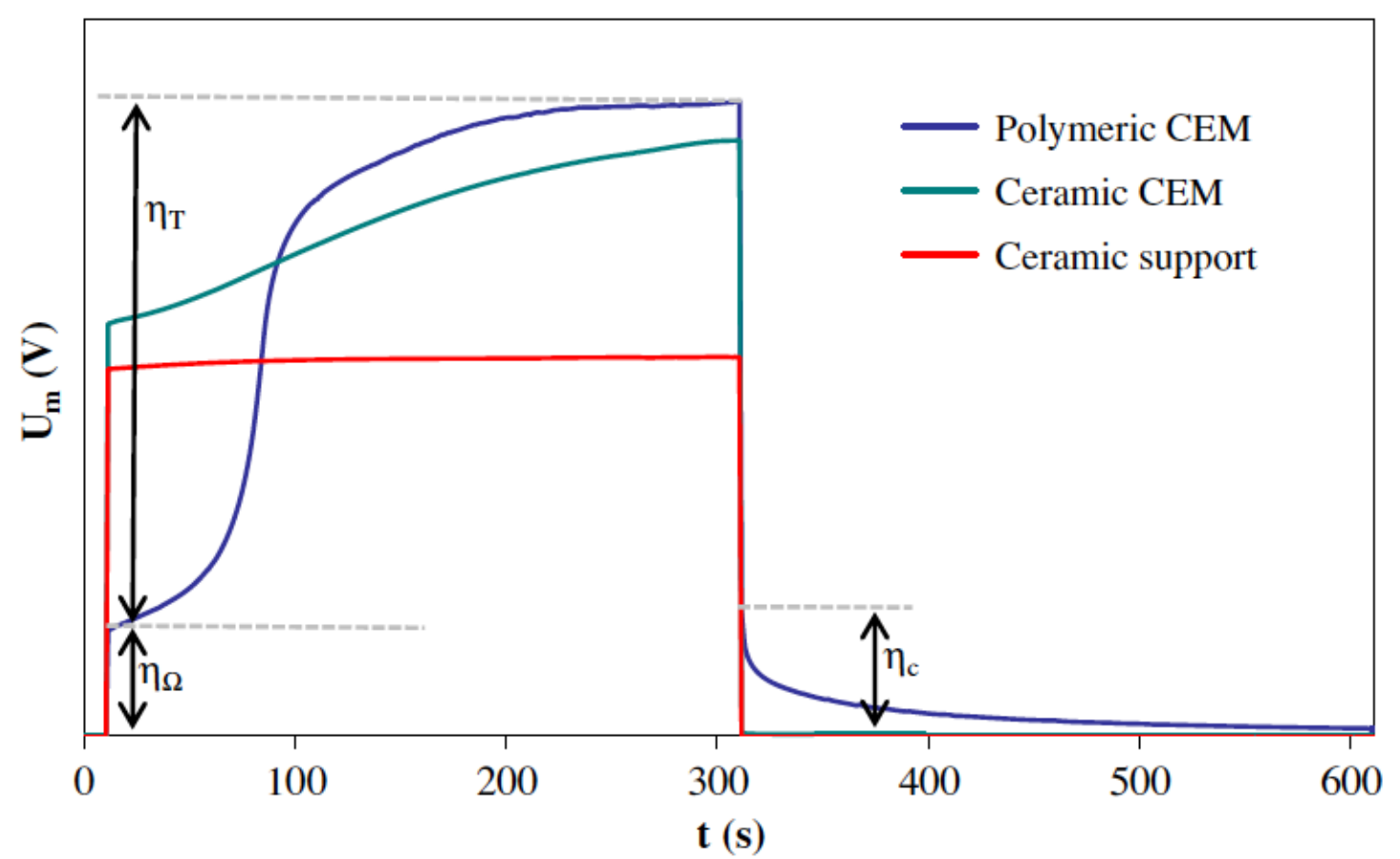

Figure 6. Comparison between the chronopotentiometric response of a commercial polymeric CEM and those of a ceramic CEM with HZrP and a ceramic support. 

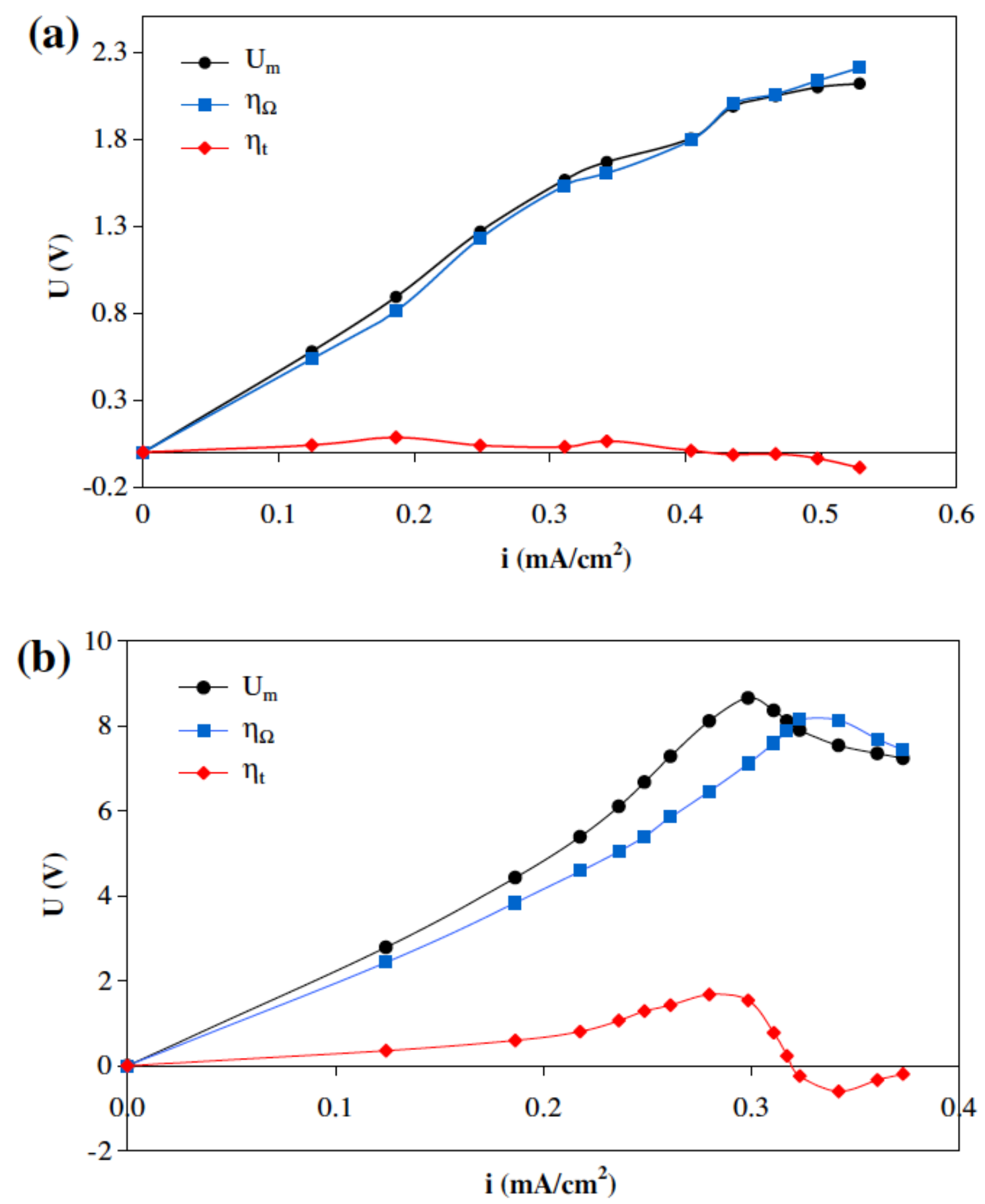

Figure 7. Representation of $U_{\mathrm{m}}, \eta_{\Omega}$, and $\eta_{\mathrm{T}}$ extracted from the chronopotentiograms obtained for $10^{-3} \mathrm{M} \mathrm{NiSO}_{4}$ solutions as a function of the applied current for the membranes with a starch content of $20 \%$ and (a) $n=0$ and (b) $n=6$. 

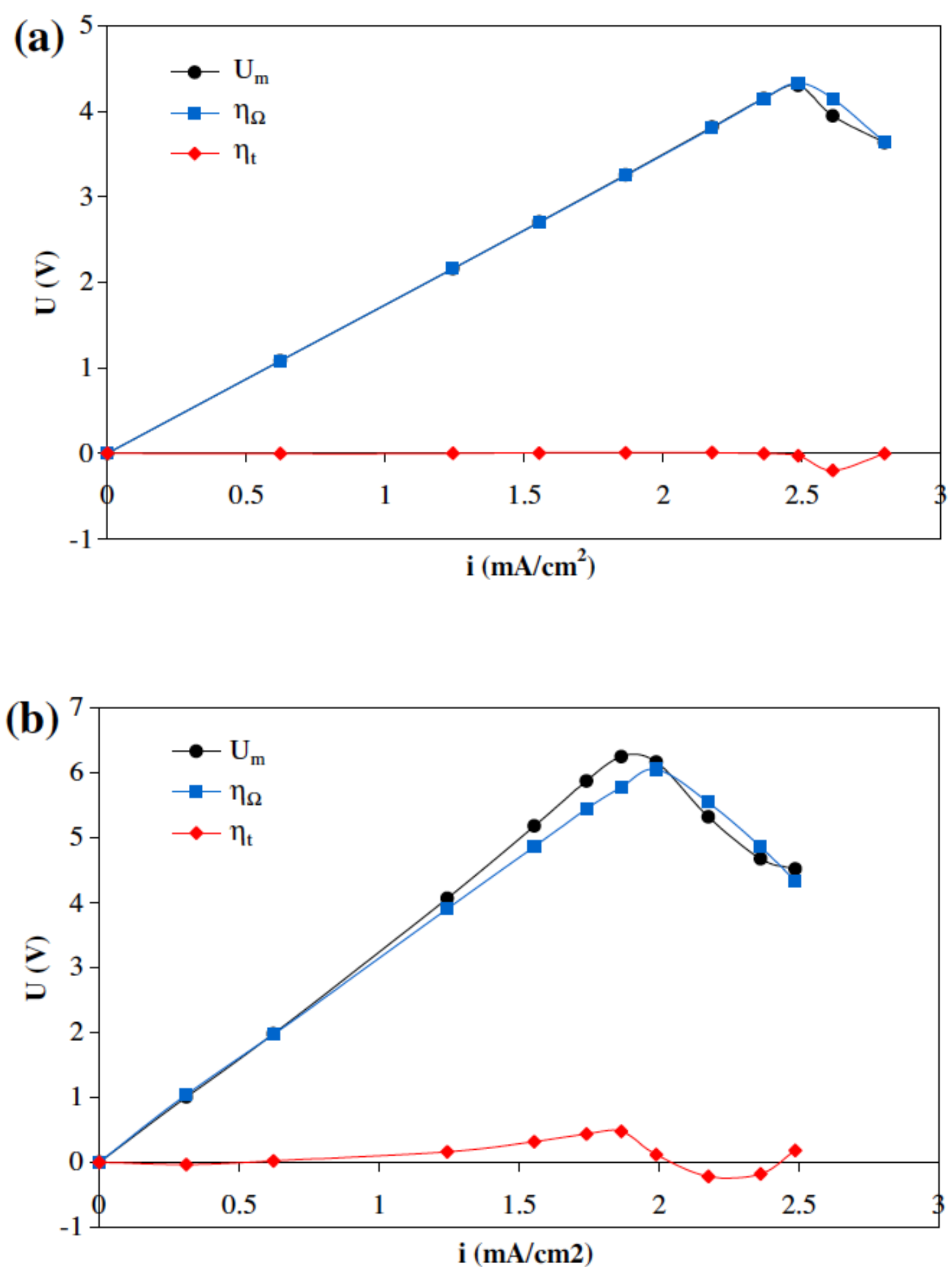

Figure 8. Representation of $\mathrm{U}_{\mathrm{m}}, \eta_{\Omega}$, and $\eta_{\mathrm{T}}$ extracted from the chronopotentiograms obtained for $10^{-2} \mathrm{M} \mathrm{NiSO}_{4}$ solutions as a function of the applied current for the membranes with a starch content of $20 \%$ and (a) $n=0$ and (b) $n=3$. 


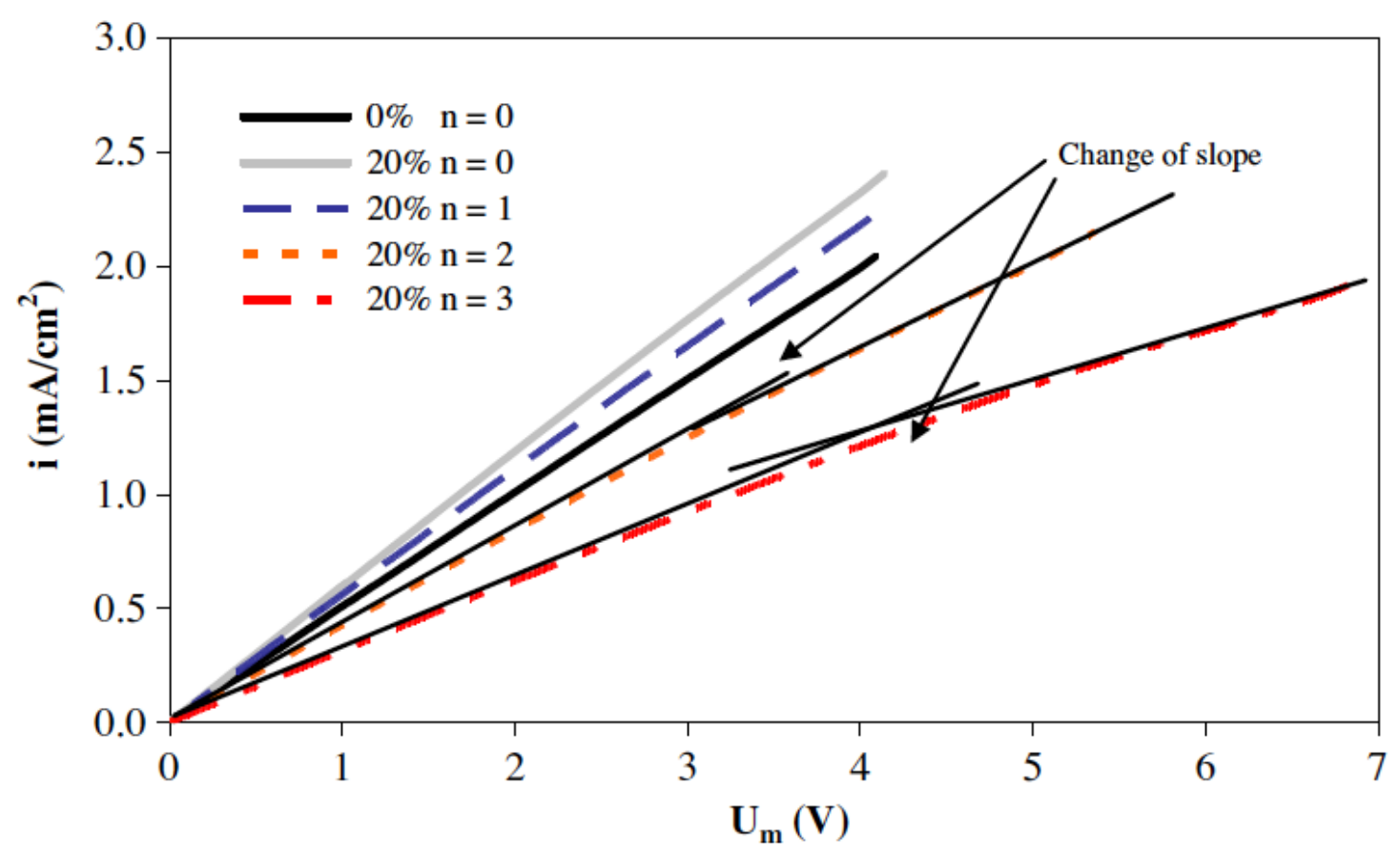

Figure 9. Current-voltage curves obtained for supports and membranes with varying number of zirconium phosphate impregnation cycles obtained in $10^{-2} \mathrm{M} \mathrm{NiSO}_{4}$ solutions.

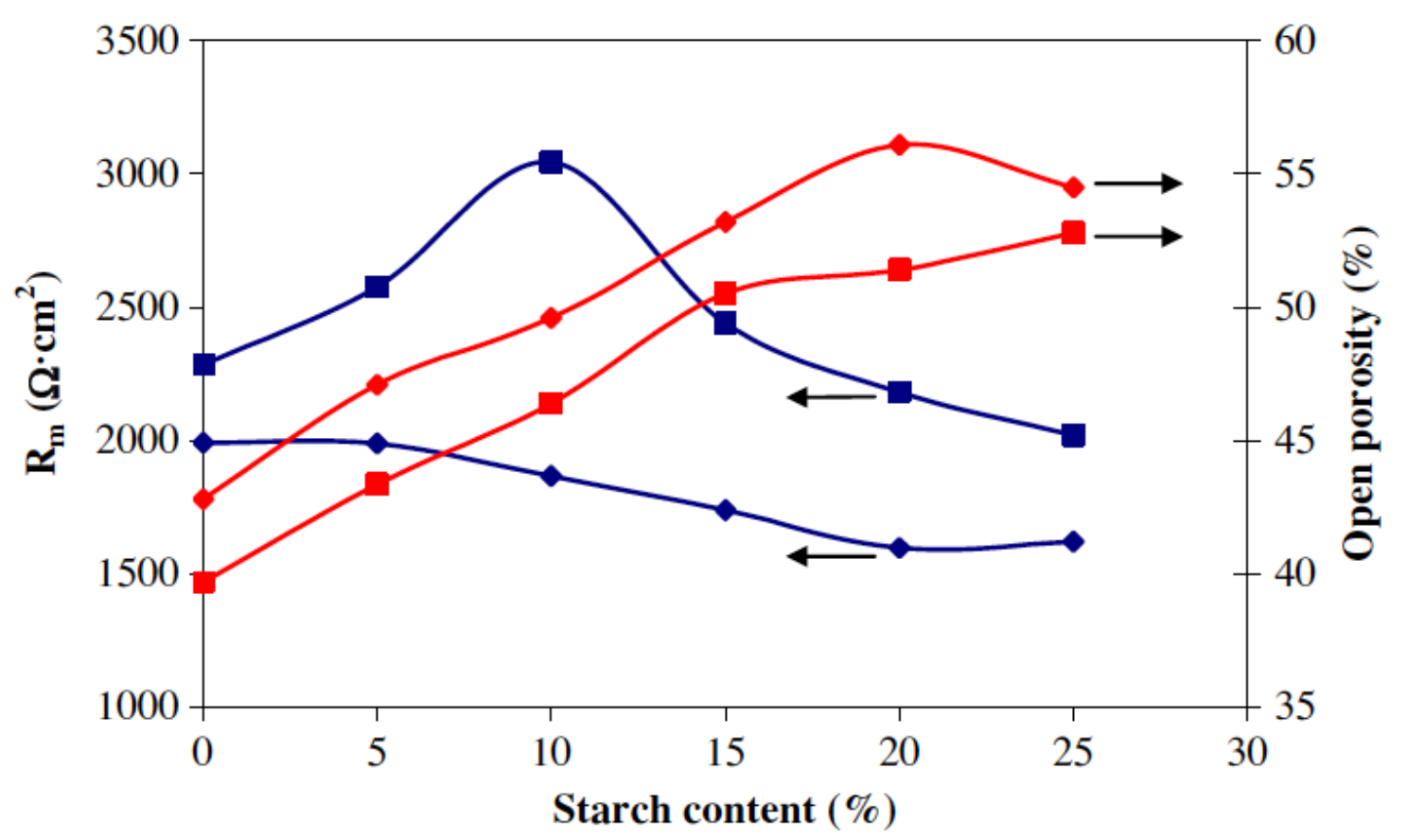

Figure 10. Resistance obtained with $10^{-2} \mathrm{M} \mathrm{NiSO}_{4}$ solutions and open porosity as a function of the starch content ( $\bullet$ supports $(\mathrm{n}=0)$ and $\mathbf{m}$ : membranes with $\mathrm{n}=1$ ). 


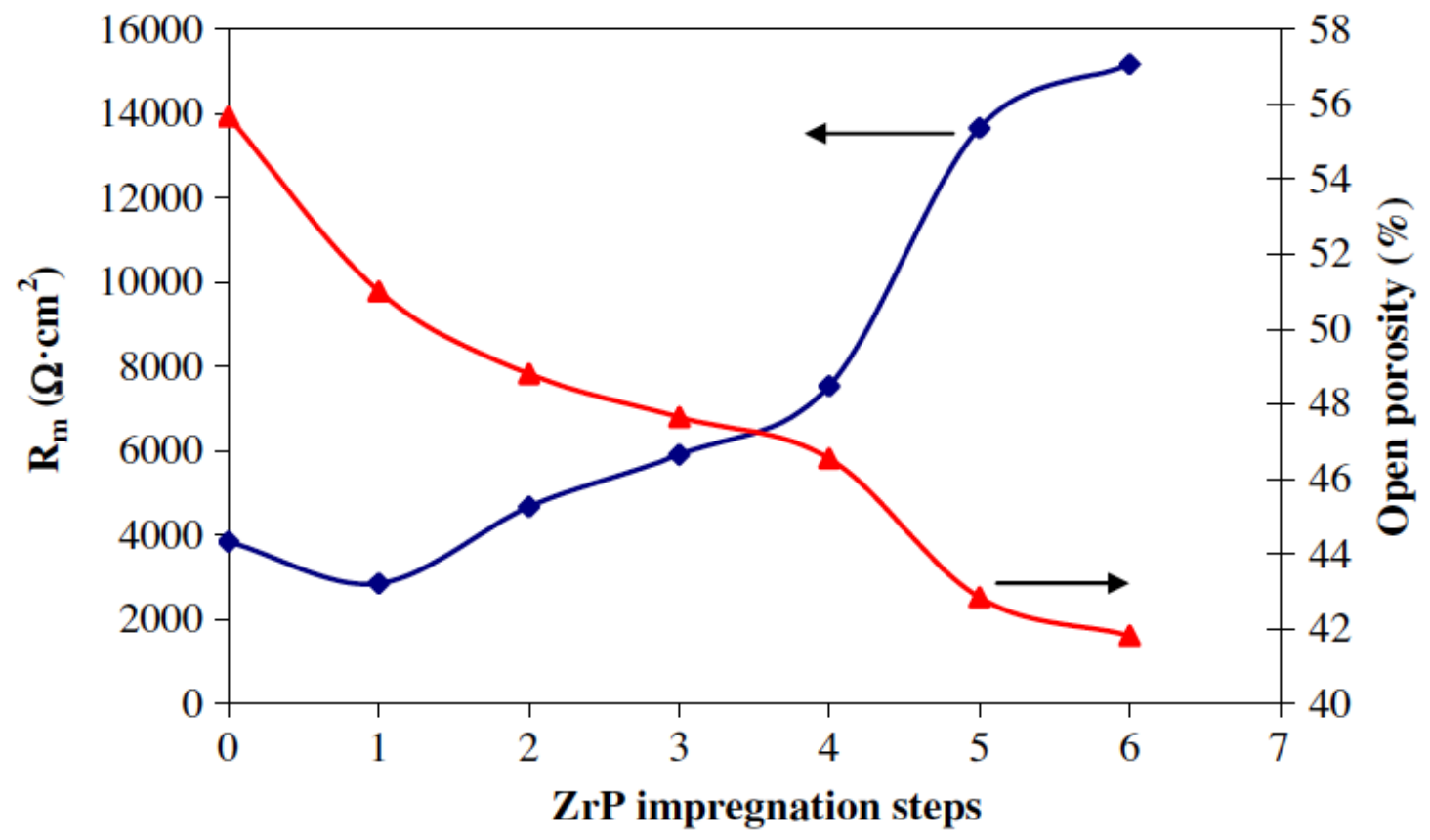

Figure 11. Membrane resistance obtained with $10^{-3} \mathrm{M} \mathrm{NiSO}_{4}$ solutions as a function of the number of impregnations. 